Check for updates

Cite this: RSC Adv., 2018, 8, 13089

\title{
Angelica gigas ameliorates the destruction of gingival tissues via inhibition of MMP-9 activity $\dagger$
}

\begin{abstract}
Mi Hye Kim, Haesu Lee and Woong Mo Yang (D)*
Angelica gigas (AG) has been used for periodontal diseases in traditional Korean medicine. However, the effects of AG on periodontitis have not been clarified yet. In this study, we investigated the ameliorative effects of AG against ligature-induced periodontitis. Sprague-Dawley rats were divided into four groups; non-ligatured (normal), ligatured and treated with vehicle (ligatured), ligatured and treated with $1 \mathrm{mg}$ $\mathrm{mL}^{-1}$ AG (AG1), and ligatured and treated with $100 \mathrm{mg} \mathrm{mL}^{-1}$ AG (AG100). 70\% ethanol extracts of AG were topically applied onto both sides of the first molar daily for 14 days. In addition, human dermal fibroblast cells were treated with 1,10 and $100 \mu \mathrm{g} \mathrm{mL}^{-1}$ AG to characterize the expression of matrix metallopeptidase 9 (MMP-9). Topical AG treatment reduced alveolar bone resorption, as assessed by methylene blue staining. The structures of soft gingival tissues (periodontal pocket) were recovered in the AG-treated groups. The expression of MMP-9 was decreased, and that of type 1 collagen was significantly increased in AG-treated gingival tissues. In addition, AG treatment inhibited the activity of MMP-9 in LPS-treated human dermal fibroblast cells. This study reveals that topical AG treatment has the potential to ameliorate the destruction of gingival tissues by inhibiting MMP-9 activity. AG may be a candidate for the treatment of periodontitis.
\end{abstract}

Received 17th November 2017
Accepted 29th March 2018

DOI: 10.1039/c7ra12531c

rsc.li/rsc-advances studies have demonstrated its pharmacological activities, which include inhibition of cancer, bacterial and nematode infection, platelet aggregation and oxidation. ${ }^{7}$ Especially, A. gigas was reported to be used as either formula or single for dental health based on the classic book. ${ }^{8}$ Previous studies demonstrate that $A$. gigas includes various chemical components such as decursin, decursinol, decursinol angelate, nodakenin, $n$-butylidenephthalide, and umbelliferoneate. Decursin has been used as a standard of identification of A. gigas, as a main bioactive compound of AG. ${ }^{9}$ Recently, decursin has the potential to inhibit ultraviolet B-induced matrix metalloproteinase (MMP)-1 and MMP-3 expression in human dermal fibroblast (HDF) cells. ${ }^{10}$ Decursin has also been shown to ameliorate bone loss by inhibiting osteoclastogenesis. ${ }^{\mathbf{1 1}}$ However, there is still a lack of study on the effects and underlying mechanism of $A$. gigas on periodontitis. In this study, we investigated the effects of $A$. gigas on periodontitis and potential mechanism in rats.

\section{Experimental}

\subsection{Preparation of AG extract}

Thirty grams of dried roots of A. gigas Nakai (Jung-do Herb; Seoul, Korea) was extracted with $300 \mathrm{~mL}$ of $70 \%$ ethanol for $24 \mathrm{~h}$ at room temperature. The extract was filtered with Whatman filter paper no. 3 (Whatman, Maidstone, Kent, England), concentrated in a rotary vacuum evaporator and freeze-dried. The weight of final dried powder (named AG) was $11.27 \mathrm{~g}$
Department of Convergence Korean Medical Science, College of Korean Medicine, Kyung Hee University, Seoul 02447, Republic of Korea. E-mail: wmyang@khu.ac.kr; Fax: +82-2-961-2209; Tel: +82-2-961-2209

$\dagger$ Electronic supplementary information (ESI) available. See DOI: 10.1039/c7ra12531c 

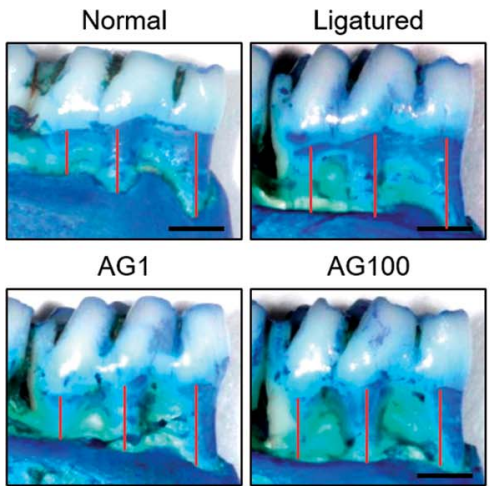

Ligatured

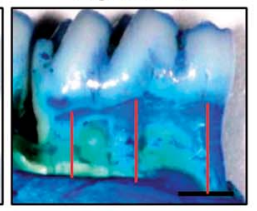

AG100
Fig. 1 Representative images and quantified values of alveolar bone loss. The scale bar is $1000 \mu \mathrm{m}$. The red lines represent the distance from the cementoenamel junction to the alveolar bone crest.

(yield: 37.6\%). A voucher specimen (AG070, 70\% ethanol extract of $A$. gigas) was deposited at our laboratory.

Decursin, which is main component of A. gigas, was used to identify AG by High-Performance Liquid ChromatographyEvaporative Light Scattering Detector (HPLC-ELSD Agilent 1100 series). The extract was dissolved in $70 \%$ methanol and sonicated $30 \mathrm{~min}$. After filtering, aliquot mixed with methanol was injected in HPLC analysis. The used column was SHISEIDO CAPCELL PAK C18 $(250 \times 4.6 \mathrm{~mm}, 5 \mu \mathrm{m})$. The mobile phase consisted of $30 \mathrm{mM}$ ammonium acetate and acetonitrile (20:80) with $1.0 \mathrm{~mL} \mathrm{~min}^{-1}$ of flow rate at $30{ }^{\circ} \mathrm{C}$. The peak of decursin in AG was synchronized with standard decursin.

\subsection{Experimental design}

Twenty-eight male Sprague Dawley rat aged 7 weeks (RaonBio Inc., Yongin, Korea) were adapted for 1 weeks in an airconditioned room under a $12 \mathrm{~h}$ light/dark cycle with food and water freely available. Appropriate temperature and humidity were maintained for animal's convenience. All experiments were approved by Committee on Care and Use of Laboratory Animals of the Kyung Hee University (KHUASP(SE)-14-029).

Twenty one rats were ligatured with sterilized 3-0 nylon into the subgingival sulcus around the both sides of first mandibular molar of rats while the rest of rats were non-ligatured, and randomly divided into 4 groups ( $n=7$, respectively); (normal) no ligature placement and non-treatment, (ligatured) ligature placement and administration of vehicle, (AG1) ligature placement and administration of AG $1 \mathrm{mg} \mathrm{mL}^{-1}$ and (AG100) ligature placement and administration of $\mathrm{AG} 100 \mathrm{mg} \mathrm{mL}^{-1}$. For reliability of topical application, $1 \%$ carboxymethylcellulose was added to AG solution in distilled water based on previous report. ${ }^{12} \mathrm{AG}$ was administered once daily in $100 \mu \mathrm{L}$ of volume at 1 and $100 \mathrm{mg} \mathrm{mL}^{-1}$ for consecutive 14 days. Then, rats were sacrificed.

\subsection{Measurement of mandible bone loss}

The right side of the mandibles were collected and immersed in $1 \%$ methylene blue aqueous solution (Sigma, MO, USA) for $1 \mathrm{~min}$ at room temperature and $20 \pm 5 \%$ humidity. After drying with compressed air, the specimens were photographed with $100 \mathrm{~mm}$ macrolens Canon digital camera (Canon, Tokyo, Japan). The length of three root surfaces from first mandibular molar were measured by a computerized densitometry system Image J (NIH, Bethesda, MD, USA). The score of alveolar bone loss was determined by sum of three values of the length of three root surfaces from first mandibular molar.

\subsection{Evaluation of histological changes}

The left side of the mandibles were fixed in $10 \%$ neutral buffered formalin for $18 \mathrm{~h}$ and demineralized in a solution of $0.1 \mathrm{M}$ ethylene diamine tetraacetic acid for 2 months. After dehydration with ethanol and xylene, the specimens were embedded in paraffin. Serial sections of $7 \mu \mathrm{m}$ thickness were obtained and stained with hematoxylin and eosin (H\&E). The digital images were obtained from Leica Application Suite (LAS) microscope software (Leica Microsystems, Buffalo Grove, IL, USA) with the $\times 40$ magnification.
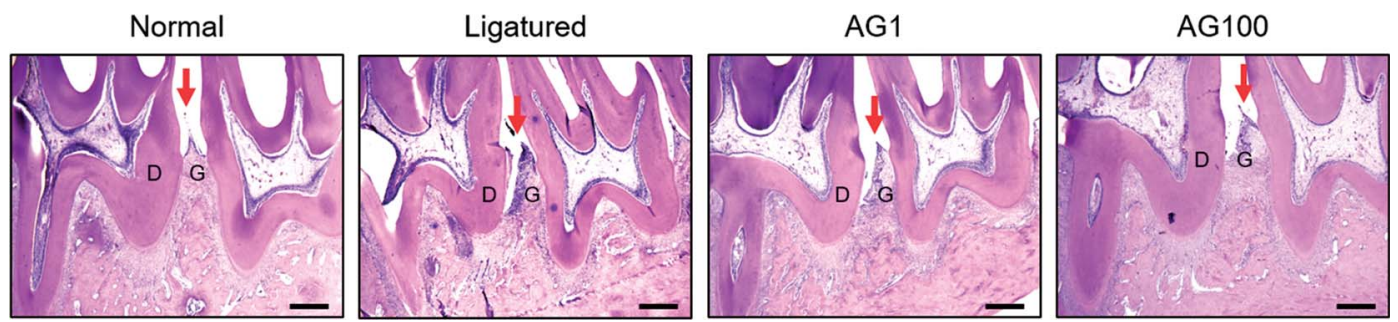

Fig. 2 Histological changes of periodontium. The scale bar is $400 \mu \mathrm{m}$. Mean values were significantly different for the following comparisons. ${ }^{\# \#} p<0.01$ compared to normal group; * $<0.05$ compared to ligatured group. Red arrows indicate to gingiva. D, dentin; G, gingiva. 
A
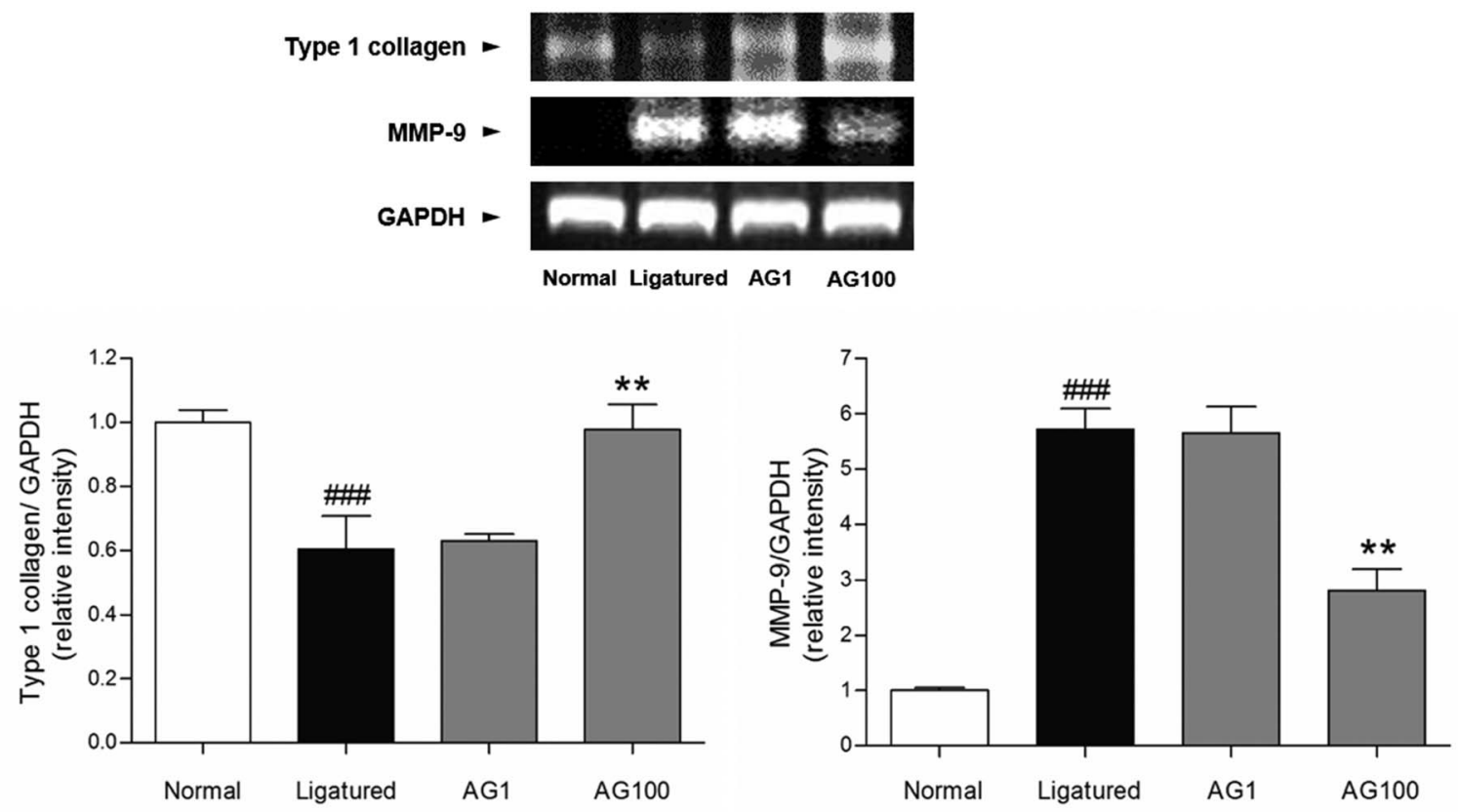

B

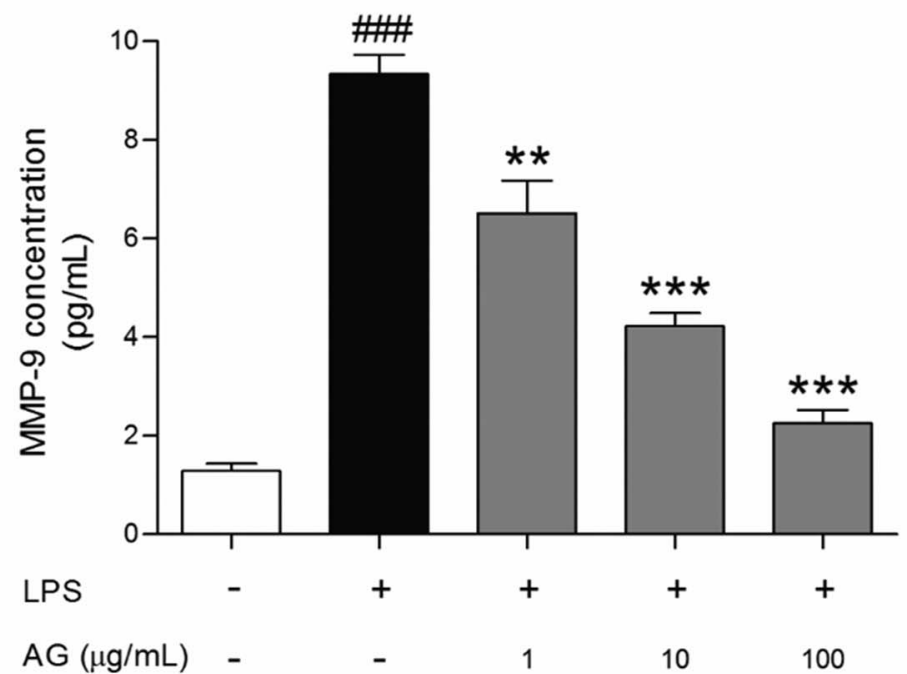

Fig. 3 Expressions of type 1 collagen and MMP-9 mRNA in gingival tissues (A). Mean values were significantly different for the following comparisons. ${ }^{\# \# \#} p<0.001$ compared to normal group; $* *<0.01$ and $* * *<0.001$ compared to ligatured group. MMP-9 concentration (pg mL ${ }^{-1}$ )

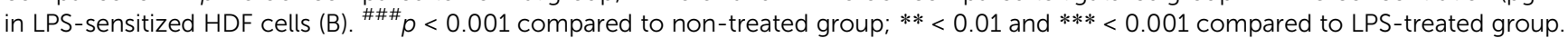

\subsection{Measurement of pro-collagen type 1 and MMP-9}

The gingival tissues around the ligature placement were excised. Total RNA from gingival tissues was extracted by Trizol methods. Complementary DNA (cDNA) was synthesized by commercially available cDNA synthesis kits (Invitrogen, Carlsbad, CA, USA) at $45^{\circ} \mathrm{C}$ for $60 \mathrm{~min}$ and then at $95^{\circ} \mathrm{C}$ for $5 \mathrm{~min}$. Reverse transcription polymerase chain reaction (RT-PCR) was performed using $10 \mu \mathrm{g}$ of cDNA with pre-mixed PCR-kit (Invitrogen). The following primers were used: type 1 collagen, $5^{\prime}$ TCT ACT GGC GAA ACC TGT ATC CG-3' (forward) and 5'-CAA GGA AGG GCA GGC GTG AT-3' (reverse). MMP-9, 5'-GGG ACG CAG ACA TCG TCA TC-3' (forward) and $5^{\prime}$-TCG TCA TCG TCG
AAA TGG GC' (reverse). GAPDH, $5^{\prime}$-CCA TCA CCA TCT TCC AGG AG $-3^{\prime}$ (forward) and $5^{\prime}$-CCT GCT TCA CCA CCT TCT TG- $3^{\prime}$ (reverse). The amplification program was comprised of the initial denaturation step 35 cycles. Relative quantification on gene expression was performed in relation to GAPDH mRNA expression by a computerized densitometry system Image J.

\subsection{Determination of MMP-9 release level}

HDF cells were grown in Dulbecco's modified Eagle's medium supplemented with $10 \%$ fetal bovine serum and $1 \%$ antibiotics (Gibco-BRL, Grand Island, NY, USA) at 5\% $\mathrm{CO}_{2}$ and $95 \%$ humidity condition. Seeded cells in 6 well plates were infected 
with $15 \mu \mathrm{g} \mathrm{mL} \mathrm{m}^{-1}$ of lipopolysaccharide (LPS) and co-treated with 1, 10 and $100 \mu \mathrm{g} \mathrm{mL} \mathrm{m}^{-1}$ of AG for $24 \mathrm{~h}$. The supernatants were collected and centrifuged. Commercial Human MMP-9 Quantikine enzyme-linked immunosorbent assay kit (R\&D systems, USA) was used to analyze the MMP-9 concentration.

\subsection{Statistical analysis}

Significance was determined by one-way analysis of variance (ANOVA) and Dunnett's multiple comparison tests using a GRAPHPAD PRISM 5 software (Ver. 5, GraphPad Software, Inc., CA, USA). In all analyses, $p<0.05$ was taken to indicate statistical significance.

\section{Results and discussion}

Periodontal disease can lead to periodontitis and gingivitis depending on the disease stage. ${ }^{13}$ Gum tissue and connective tissue becomes inflamed (gingivitis) at early stage, and further advances to periodontitis such as alveolar bone loss. ${ }^{1}$ Accordingly, recent therapies for periodontal disease are required to target not only alveolar bone resorption, but also soft tissue degradation. ${ }^{14}$ In the present study, a significant increase of alveolar bone loss was appeared (normal $=2.495 \pm 0.34 \mathrm{~mm}$; ligatured $=3.338 \pm 0.172 \mathrm{~mm}$ ) in ligature-induced periodontitis group. Compared to ligatured group, AG $100 \mathrm{mg} \mathrm{mL}^{-1}$ treated group showed a significant decrease in alveolar bone loss $(\mathrm{AG} 1=3.203 \pm 0.167 \mathrm{~mm} ; \mathrm{AG} 100=2.925 \pm 0.275 \mathrm{~mm} ; p<0.05$, Fig. 1). In addition, severe destruction of gingival tissues in space between first and second mandibles regarded as periodontal pocket was observed in ligatured group in comparison with normal group. Treatment of 1 and $100 \mathrm{mg} \mathrm{mL}^{-1}$ of AG recovered the collapsed gingival tissues compared with ligatured group (Fig. 2). Especially, $100 \mathrm{mg} \mathrm{mL}^{-1}$ of AG-treated group showed an almost complete recovery nearby normal group. Taken together, AG treatment ameliorated the formation of periodontal pockets resulting from soft tissue destruction, as well as alveolar bone loss, in a ligature-induced periodontitis rat model.

Fibroblasts, which produce a collagen-rich extracellular matrix, are the predominant components of gingival tissues. ${ }^{15}$ Well-organized type 1 collagen is responsible for the adherence of the gingiva to the teeth. ${ }^{16}$ It is well established that gingival inflammation (i.e., gingivitis) accompanies the development of periodontitis. In periodontitis, the destruction of gingival tissues results in the degradation of collagen, including type 1 collagen. ${ }^{17}$ AG treatment recovered the destruction of gingival tissue, which is affected by periodontitis. These improvement of soft tissue inflammation was accompanied with an increase of type 1 collagen mRNA expression by AG treatment. Gingival tissue from ligatured group exhibited a significant lower mRNA expression of type 1 collagen $(p<0.05)$ compared with normal group. The mRNA expressions of type 1 collagen were increased dose-dependently by AG treatment (Fig. 3A).

To clarify the protective effects of AG on collagen degradation, collagenase activity was investigated in gingival tissue. MMPs are responsible for the degradation of denatured interstitial collagens in damaged tissue. ${ }^{18-20}$ As described in previous reports, MMP-9 distributed in fibroblasts, keratinocytes, endothelial cells and osteoblasts could be a key enzyme to disassemble the type 1 collagen. Therefore, inhibition of MMP-9 activity might be involved in ability to attenuate the degradation of gingival tissues and the expression of MMP-9 is seriously increased in impaired gingiva by periodontal inflammation. ${ }^{21}$ The present study shows that the activity of MMP-9 in gingival tissue was significantly inhibited by AG treatment. The mRNA expression of MMP-9 was clearly increased in ligatured group. Treatment of AG $100 \mathrm{mg} \mathrm{mL}^{-1}$ reduced the mRNA expression of MMP-9 (Fig. 3A). To confirm the effect on MMP-9 expression by AG treatment, collagenase activity was analyzed in vitro. Approximately 7.25 times of increase of MMP-9 level was found in LPS-induced HDF cells. Following treatment with 1, 10 and $100 \mu \mathrm{g} \mathrm{mL} \mathrm{m}^{-1}$ of AG, elevated MMP-9 concentration were

\section{Gingival tissue}

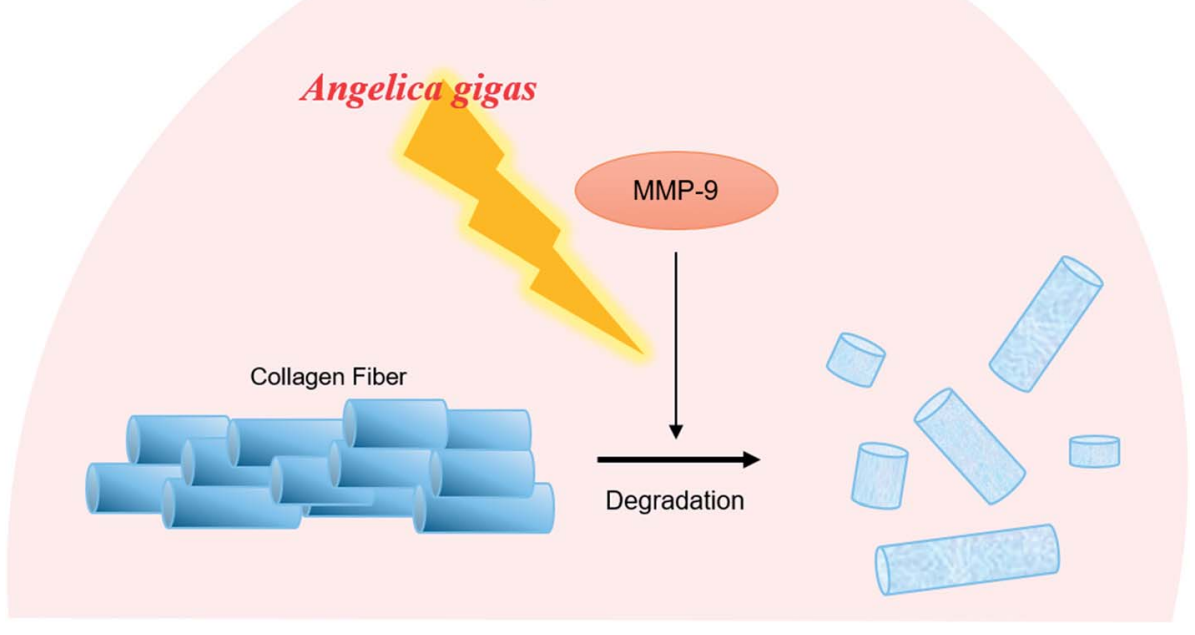

Fig. 4 Diagram illustrates the potential actions of AG on destruction of gingival tissues in periodontitis. 
suppressed in dose-dependent manner (30.22, 54.84 and $75.83 \%$, respectively, Fig. 3B). These results suggest that the restoration of gingival tissue by AG treatment is positively correlated with the inhibition of MMP-9 activity.

\section{Conclusion}

In conclusion, topical AG treatment inhibited the alveolar bone loss in ligature-induced periodontitis rat. AG improved the periodontal pocket formation by recovery of the destruction of gingival tissues. The expressions of type 1 collagen and MMP-9 in gingival tissue were significantly regulated by AG administration. The present study revealed that topical AG treatment ameliorates alveolar bone loss and gingiva tissue degradation by inhibiting MMP-9 activity (Fig. 4). These results suggest that AG may help regenerate impaired gingiva in periodontitis.

\section{Conflicts of interest}

The authors declare no conflict of interest.

\section{Acknowledgements}

This work was supported by the National Research Foundation of Korea (NRF) grant funded by the Korea government (MSIP) (NRF-2017R1A6A3A11032500 and 2016R1D1A2B03935368).

\section{References}

1 S. Offenbacher, Annals of periodontology, 1996, 1, 821-878.

2 R. C. Williams, A. H. Barnett, N. Claffey, M. Davis, R. Gadsby, M. Kellett, G. Y. Lip and S. Thackray, Curr. Med. Res. Opin., 2008, 24, 1635-1643.

3 T. H. Howell and R. C. Williams, Crit. Rev. Oral Biol. Med., 1993, 4, 177-196.

4 P. M. Preshaw, A. F. Hefti, S. Jepsen, D. Etienne, C. Walker and M. H. Bradshaw, J. Clin. Periodontol., 2004, 31, 697-707.
5 H. Yang, Q. Wen, J. Xue and Y. Ding, J. Periodontal Res., 2014, 49, 382-389.

6 J. H. Cho, J. E. Kwon, Y. Cho, I. Kim and S. C. Kang, Nutrients, 2015, 7, 4862-4874.

7 B. Lee, B. Sur, I. Shim, H. Lee and D. H. Hahm, BMC Complementary Altern. Med., 2015, 15, 210.

8 B. K. Song, J. H. Won and S. Kim, J. Pharmacopuncture, 2016, 19, 16-20.

9 S. Y. Jeong, H. M. Kim, K. H. Lee, K. Y. Kim, D. S. Huang, J. H. Kim and R. S. Seong, Chem. Pharm. Bull., 2015, 63, 504-511.

10 B. M. Hwang, E. M. Noh, J. S. Kim, J. M. Kim, J. K. Hwang, H. K. Kim, J. S. Kang, D. S. Kim, H. J. Chae, Y. O. You, K. B. Kwon and Y. R. Lee, Int. J. Mol. Med., 2013, 31, 477-483.

11 X. Wang, T. Zheng, J. H. Kang, H. Li, H. Cho, R. Jeon, J. H. Ryu and M. Yim, Eur. J. Pharmacol., 2016, 774, 34-42.

12 M. H. Kim, Y. Y. Choi, H. J. Lee, H. Lee, J. C. Park and W. M. Yang, J. Periodontal Implant Sci., 2015, 45, 145-151.

13 J. J. Reynolds and M. C. Meikle, Periodontology 2000, 1997, 14, 144-157.

14 P. M. Bartold, M. D. Cantley and D. R. Haynes, Periodontology 2000, 2010, 53, 55-69.

15 G. J. Fisher, S. C. Datta, H. S. Talwar, Z. Q. Wang, J. Varani, S. Kang and J. J. Voorhees, Nature, 1996, 379, 335-339.

16 F. Trindade, F. G. Oppenheim, E. J. Helmerhorst, F. Amado, P. S. Gomes and R. Vitorino, Proteomics: Clin. Appl., 2014, 8, 748-761.

17 H. Hatipoglu, F. Yaylak and Y. Gungor, Diabetes, Metab. Syndr. Obes.: Targets Ther., 2015, 9, 124-126.

18 C. M. Cobb, J. Clin. Periodontol., 2002, 29, 6-16.

19 P. C. Smith, V. C. Munoz, L. Collados and A. D. Oyarzun, J. Periodontal Res., 2004, 39, 87-92.

20 J. Santos, V. D. La, C. Bergeron and D. Grenier, J. Periodontal Res., 2011, 46, 550-557.

21 M. Makela, T. Salo, V. J. Uitto and H. Larjava, J. Dent. Res., 1994, 73, 1397-1406. 